\title{
Two Novel, Simple-Structured and Easy-Synthesized Acceptors Based on Fluorene or Carbazole for Non- Fullerene Organic Solar Cells
}

\section{Fubiao Weng}

Xiangtan University

Songping Shen

Xiangtan University

Peijin Zheng

Xiangtan University

Gengbiao Xu

Xiangtan University

Zhiwei Qiu

Dongguan University of Technology

Jianhao Chen

Dongguan University of Technology

Yongtao Chen

Dongguan University of Technology

shengming peng ( $\nabla$ smpeng111@163.com )

Xiangtan University https://orcid.org/0000-0002-0205-1560

Junxu Liao

Dongguan University of Technology

Hongbin Zhao

Dongguan University of Technology

\section{Research Article}

Keywords: OSCs, carbazole, fluorene, deep HOMO, PTB7-Th

Posted Date: May 24th, 2021

DOI: https://doi.org/10.21203/rs.3.rs-550529/v1

License: (1) (1) This work is licensed under a Creative Commons Attribution 4.0 International License.

Read Full License 


\section{Abstract}

Two novel D- $\pi-A-\pi-D$ type non-fullerene acceptors (FPTC and CPTC) composed of fluorene or carbazole as acceptor unit, benzene as intermediates and 2-(6-oxo-5,6-dihydro-4H-cyclopenta[c] thiophen-4-ylidene) malononitrile (TC) as terminal groups are synthesized through only two procedures. Also, their electrochemical behavior, photophysical properties and photovoltaic performance are systematically characterized and thoroughly studied. In consequence, the FPTC has better performance than C PTC, and the PCE of this device based on FPTC: PTB7-Th is nearly $1 \%$ higher than that of CPTC: PTB7-Th device, reaching up to $1.09 \%$ with a V OC of $0.71 \mathrm{~V}$, a J SC of $3.42 \mathrm{~mA} \mathrm{~cm}-2$. The higher PCE of the device based on FPTC is attributed to the fact that this molecular has a wider absorption spectrum and a higher molar extinction coefficient nearly four times than that of CPTC, a higher initial oxidation potential, and a lower onset reduction potential. Also, the higher electron mobility rate and hole mobility rate contribute to the device based on FPTC better performances. Compared with carbazole, the fluorene as the acceptor unit provides potential possibilities for the construction of high-performance organic solar cells.

\section{Introduction}

In the past few decades, Organic solar cells (OSCs) have attracted a great deal of attention for their visible advantages such as low-cost manufacturing, simple manufacturing process, material diversity, modifiable structure, great flexibility, solution processability which has been known as one of the most promising materials to generate electricity in a green and environmentally friendly ${ }^{1-4}$. It is well known that organic solar cells (OSCs) are composed of electron-sucking acceptors and electron-donators. The electron acceptors based on fullerene derivatives once have Has brought a research upsurge to researchers for isotropic charge transport and favorable nanoscale network forming behaviors which made its power conversion efficiency (PCE) reached more than $11.3 \%{ }^{4}$. However, the acceptor of fullerene derivatives still has many defects that make it not widely used in high-efficiency organic solar cells. In particular, the compound with two organic cyclic adducts is randomly distributed on the fullerene cage, which means that the compound has multiple stereoisomers and even the structure is uncertain, resulting in considerable large structure and location disorder, Therefore, this phenomenon inhibits charge transfer leads to their poor photovoltaic performance ${ }^{5-8}$, In recent years, owning to well-determined molecular structure and weight, tunable molecular energy levels, low-cost synthesis for easier purification, better repeatability of device, potential of industrial production for low batch-to-batch variation, increasing number of researchers have invested more efforts to study non-fullerene acceptors(NFAs) which have obviously advantages different from fullerene receptors ${ }^{3,9-11}$. Today, with the continuous improvement of materials, innovation device engineering and morphological optimization, the photovoltaic performance based on non-fullerenes has already surpassed that of fullerenes derivatives, with PCE of more than $18 \%$. In consequence, the design and synthesis of NFAs provides a new opportunity for the commercial application of OSCs. 
Small molecule organic solar cells (SM-OSCs) and polymer solar cells (PSCs) are consisted of Nonfullerene organic solar cells. Nowadays, although the performance of the OSCs based small molecule still lags behind that of polymer, the SM-OSCs have their unique advantages such as well-determined and design structure, easier synthesis with higher purification, solution-processed, intermolecular arrangements characterized by crystallographic analysis, which attracts increasing attention from researcher $^{2,12}$. In SM-OSCs, combining electron-rich groups and electron-deficient groups as the efficient electron acceptors is the most common design method in order to obtain efficient intermolecular charge transfer (ICT) and low energy gap, which enable to broaden the absorption spectrum and promote the transition or separation of electrons from excited molecules to acceptors. According to reports, the D- $\pi-A-$ $\pi-D$ A- $\pi-A, A-\pi-D, D-\pi-D$ and D-A-D conjugated structures are commonly applied in small organic molecules. the A- $\pi-A, A-\pi-D, D-\pi-D$ and $D-A-D$ conjugated structures have many inevitable problems such as ladder-type polycyclic compound containing strongly deficient electrons and rich electrons group involves complex synthesis and multi-step purification, which immensely increases the cost of material and synthesis. However, D- $\pi-A-\pi-D$ type is the most attractive conjugated structures, because its photovoltaic performance modified by electron-rich donors, electron-deficient acceptors, and $\pi$ bridges and its $3 D \pi$-conjugated conformations provides high electron cloud density to improve charge delocalization, which helps reduce the energy level gap ${ }^{2,13}$.

In this work, we designed and synthesized two small molecules based on fluorene and carbazole as acceptors for bulk heterojunction (BHJ) SM-OSCs. The design of this molecule is based upon the following consideration: $\mathbb{~}$ ) With the planarization of biphenyl units, fluorene is a kind of electron-rich rigid conjugated molecule similar to a spiro ring. which can decrease the oxidation potential, show great thermal photochemical, thermal stability and low lying lowest unoccupied molecular orbital (LUMO), The fluorenyl moiety can cause a highly sterically hindered structure that has a huge impact on the solubility of the entire molecule ${ }^{13-19}$. 『) Carbazole has a low-lying Highest Occupied Molecular Orbital (HOMO) level and only one substitution position (N-position) which can be expected the $\pi-\pi$ stacking distance between molecules ${ }^{20-23}$. 『) 2- (6-oxo-5,6-dihydro-4H-cyclopenta [c] thiophen-4-ylidene) malononitrile (TC) was an excellent end group for acceptor moiety for its strong light-harvesting from the electron

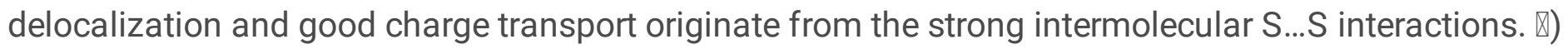
Benzene is an electron-rich molecule with a conjugate plane and is a common structural unit used as $\pi-$ bridge in organic optoelectronic materials.

Herein, according to these factors, we synthesized two novel non-linear D- $\pi-A-\pi-D$ type small molecule FPTC and CPTC (Fig. 1) acceptors that bear the same 2-(6-oxo-5,6-dihydro-4H-cyclopenta[c]thiophen-4ylidene) malononitrile(TC) as acceptor moiety, fluorene or carbazole as donor moiety and benzene as $\pi-$ bridge for FPTC and CPTC, respectively. The synthesis of the target molecule only passes through two steps and the yield exceeds $80 \%$, which may be provide the possibility of the potential application of large-scale production. Both compounds have great solubility in common organic solvents, such as dichloromethane, chloroform, tetrahydrofuran (THF), and chlorobenzene (CB) at room temperature. To greatly match energy levels and widen complementary absorption spectra, we have treated FPTC and 
CPTC as a donor component along with poly[(4,8-bis(5-(2-ethylhexyl) thiophen-2-yl)benzo[1,2-b:4,5b']dithiophene2,6-diyl)-alt-(2-ethylhexyl-3-fluorothieno [3,4-b]thiophene-2 carboxylate-4,6-diyl)] (PTB7-Th) as donor to fabricate the inverted bulk-heterojunction organic solar cells (BHJ-OSCs). Also, in this work, we utilized a great deal of method to characterized these SMs structure, optical and electrochemical properties, morphology and photovoltaic parameters, which could deeply understand the structure property relationships and provide strategy to construct this type compound. Finally, the FPTC has better performance than CPTC, and the PCE of this device based on FPTC: PTB7-Th is nearly $1 \%$ higher than that of CPTC: PTB7-Th device, reaching up to $1.09 \%$ with a $V_{O C}$ of $0.71 \mathrm{~V}, \mathrm{a} J_{S C}$ of $3.42 \mathrm{~mA} \mathrm{~cm}{ }^{-2}$. The higher PCE of the device based on CPTC: PTB7-Th is attributed to the fact that this device has a wider absorption spectrum and a molar extinction coefficient nearly four times higher than that of FPTC: PTB7Th device, a higher initial oxidation potential, and a lower onset reduction potential. Also, the higher electron mobility rate and hole mobility rate contributes to its better performances. By changing the central core, we have a great understand that fluorene core is a good strategy for constructing high performance SMAs.

\section{Results And Discussion}

\subsection{Materials synthesis and characterization}

The synthetic routes of FPTC and CPTC are demonstrated in Scheme 1. Both of two target molecules were synthesized from readily available and inexpensive raw materials which used without any further purification. Only two steps are needed to synthesize these acceptors with a yield on top of $80 \%$. 2,2'-(9(heptadecan-9-yl)-9H-fluorene-2,7-diyl)bis(4,4,5,5-tetramethyl-1,3,2-dioxaborolane) (1) and 9-(heptadecan9-yl)-2,7-bis(4,4,5,5-tetramethyl-1,3,2-dioxaborolan-2-yl)-9H-carbazole (5) were prepared according to the per reported literature. The intermediates 3 and 6 were synthesized via the Suzuki coupling reaction of compounds 1 or 5 with 4 -bromobenzaldehyde (2) at $110^{\circ} \mathrm{C}$ in over $90 \%$ yield.

The reaction of intermediates 3 with 2-(6-0xo-5,6-dihydro-4H-cyclopenta[c]thiophen-4ylidene)malononitrile in chloroform (4) at $25{ }^{\circ} \mathrm{C}$ resulted FPTC in good yield of $84.2 \%$. Similar to the synthesis method of target compound FPTC, the reaction of intermediates 3 with molecule 4 at 25 ${ }^{\circ}$ Cachieved CPTC in good yield of $81.5 \%$. All molecules structure was characterized by MALDI-TOF-MS, ${ }^{1} \mathrm{H}$ Nuclear Magnetic Resonance ( ${ }^{1} \mathrm{H}$ NMR) and ${ }^{13} \mathrm{C}$ NMR and the synthetic details and characterization data are presented in the Supporting Information.

The new acceptors FPTC and CPTC have excellent solubility in common solvents such as dichloromethane, toluene, chlorobenzene, tetrahydrofuran, chloroform and chlorobenzene at ambient temperature, which means that they have the potential of large scale production via processing solvents in the field of photovoltaic applications. The thermal stabilities of FPTC and CPTC were characterized by thermogravimetric analysis (TGA), and it shows that the decomposition temperature $\left(T_{d}\right.$, corresponding to a $5 \%$ weight loss) of FPTC and CPTC is $212^{\circ} \mathrm{C}$ and $173^{\circ} \mathrm{C}$ under inert atmosphere (Fig. S1), respectively, which proves that thermal decomposition temperature of FPTC is nearly 40 degrees Celsius 
higher than that of CPTC and it means that FPTC bearing fluorene as central core have greater thermal stabilities and more feasible for vacuum-deposition fabrication.

\subsection{Optical and electrochemical properties}

The UV-Vis absorption spectra of the molecule of FPTC and CPTC in chloroform solution and thin film form are shown in Fig. 2a-2b. Relevant optical parameters have recorded in Table 1. Both of FPTC and CPTC demonstrate a wide and intense absorption that extends from 350 to $650 \mathrm{~nm}$. The broad absorption suggests strong intramolecular charge transfer (ICT) actively from donor to acceptor moieties. In the neat film, for CPTC and FPTC, both display broader absorption spectra and significantly red-shifted absorption spectra than in solution, with the maximum absorption peaks at $515 \mathrm{~nm}$ and $517 \mathrm{~nm}$, respectively. Compared to CPTC, FPTC exhibits distinct peaks in the wavelength of $498 \mathrm{~nm}$ with the highest molar extinction coefficients of $6.64 \times 10^{4} \mathrm{M}^{-1} \mathrm{~cm}^{-1}$, whereas CPTC just gave a relatively faint vibronic peak in $486 \mathrm{~nm}$ and its highest molar extinction coefficients only have $1.75 \times 10^{4} \mathrm{M}^{-1} \mathrm{~cm}^{-1}$, which should be attributed to the fact that FPTC has stronger intermolecular p-p, $\pi-\pi$ stacking and mutual conjugation than CPTC. Owing strong intermolecular interactions in the solid state, the absorption peak of FPTC is red shifted around $30 \mathrm{~nm}$ from the solution to the solid state. However, the interesting phenomenon is that the maximum absorption peaks of CPTC in film is $513 \mathrm{~nm}$ with the edge absorption wavelength of $643 \mathrm{~nm}$, which is red-shifted by $27 \mathrm{~nm}$ compared with that in solution, and even $10 \mathrm{~nm}$ longer than FPTC, possibly as a result of the effect of the intramolecular non-covalent $\mathrm{N} \cdots \mathrm{H}, \mathrm{N} \cdots \mathrm{O}$ and $\mathrm{O} \cdots \mathrm{H}$ interactions, intermolecular $\pi-\pi$ bond interactions and effective solid stacking. Also, the absorption edge is red-shifted, suggesting that more acceptor moieties possibly enlarge the energy gap for intramolecular electron transition.

Contrast to the molar extinction coefficient, we can infer that all of them have a relatively high molar extinction coefficient, indicating that the donor units effectively enhanced the absorption when the fluorene and carbazole was used as a central core, respectively. The molar extinction coefficient of FPTC is about 3.8 times that of CPTC which means that FPTC has a better ability to capture photons due to the best planarity of fluorene central core and the strongest ICT effect, and finally increase the Jsc of OSCs.

The electrochemical properties of FPTC and CPTC were examined by the cyclic voltammetry (CV) in $\mathrm{CH}_{3} \mathrm{CN} / 0.1 \mathrm{M} \mathrm{Bu}_{4} \mathrm{NPF}_{6}$ solution at a scan rate of $100 \mathrm{mV} / \mathrm{s}$ with ferrocene/ferrocenum $\left(\mathrm{Fc} / \mathrm{Fc}^{+}\right)$redox couple (4.8 eV under the vacuum level) as the internal calibration. The CV curves are shown in Fig. $2 \mathrm{c}$ and its corresponding detail results are collected in Table 1. According to the CV curves, we can inform that the $\mathrm{E}_{\mathrm{OX} \text { onset }}$ (onset oxidation potential) of SMAs are observed as $1.30 \mathrm{eV}$ and $1.25 \mathrm{eV}$, respectively. Then, with the equation of $E_{R E \text { onset }} E_{O X}$ onset ${ }^{-}$Eopt $g$, we can easily calculated the value of the $E_{R E}$ onse (onset reduction potential), corresponding to $-0.40 \mathrm{eV}$ and $-0.32 \mathrm{eV}$, which is almost in agreement with the experimental values obtained from reduction waves. Also, according to the following equation: $\mathrm{E}_{\mathrm{HOMO}}=[(-$ $\left.\left.E_{\text {OX onset }}-0.52\right)-4.8\right] \mathrm{eV}, \mathrm{E}_{\mathrm{LUMO}}=\left[\left(-\mathrm{E}_{\text {red onset }}-0.52\right)-4.8\right] \mathrm{eV}$ and $\mathrm{E} \mathrm{g}=\mathrm{E}_{\mathrm{HOMO}}-\mathrm{E}_{\mathrm{LUMO}} \mathrm{eV}$, the HOMO and LUMO energy levels of SMAs are calculated to be as $-5.75 /-4.05$ and $-5.70 /-4.17 \mathrm{eV}$. respectively. All the 
SMAs have deep HOMO/LUMO levels and higher $\Delta \mathrm{E}_{\mathrm{HOMO}} / \Delta \mathrm{E}_{\mathrm{LUMO}}$, which ensure the exciton dissociate and transfer from donor to acceptor, implying that they could be utilized as electron acceptor materials in the SM-OSCs. In contrast, we can find that the CPTC based carbazole core have lower LUMO ( $-4.05 \mathrm{eV}$ vs $-4.13 \mathrm{eV})$ and higher HOMO level ( $-5.75 \mathrm{eV}$ vs $-5.70 \mathrm{eV}$ ) that applying carbazole as acceptor moiety can effectively improve their HOMO/ LUMO energy levels for the electron-withdrawing effect of $\mathrm{N}$ atom. Based on the principle of mutual energy level matching and complementary light absorption, we decide PTB7-Th (-5.22/-3.64 eV) as donor and our molecules as acceptor. The energy diagram relative to the vacuum level were shown in Fig.1d. The LUMO-LUMO offset of two acceptors and PTB7-Th are 0.41 $0.49 \mathrm{eV}$, and all of them exceed $0.3 \mathrm{eV}$ which is enough for efficient exciton dissociation and charge separation at the donor-acceptor interface. What's the most important is that the HOMO energy offset ( $\mathrm{E}_{\text {HOMO }}$ ) between PTB7-Th and the acceptors are 0.48 and $053 \mathrm{eV}$, respectively, which enable efficient hole transfer from the acceptors the PTB7-Th to donor.

\subsection{Computational analysis}

The theoretical calculations of density functional theory (DFT) was conducted by Gaussian 09 package57 at the B3LYP/631G(d) level to investigate the influence of molecular structure and electronic distribution on absorption spectra and electrochemical performance ${ }^{24}$. The optimized molecular structures and frontier molecular orbitals of the two acceptors are shown in Figure 3a-b, corresponding detail parameters are summarized in Table 2. In the optimized molecular structures of FPTC and CPTC, both of their structures are symmetrical and the dihedral angles on the left and right sides are basically the same. We record $\theta$ as the dihedral angle. The $\pi$-bridged phenyl group shows a large dihedral angle with two acceptor moieties or with terminal groups, forming a non-planar structure. This must seriously affect the aggregation state of the molecules. In contrast, the SMAs have large dihedral angle between phenyl bridges and terminal TCs reaching up to $90^{\circ}$. The dihedral angle between the central donor unit carbazole and the adjacent $\pi$-bridge phenyl group is approximately $18^{\circ}$, which the dihedral angle between the central fluorene and adjacent phenyl bridge is only $16^{\circ}$. It means that the presence of carbazole unit intensify the conjugation of the molecule, but it is not conducive to the coplanarity of the whole molecules due to the angle with the $\pi$-bridge and intramolecular steric hindrance, which prevents the transfer of intramolecular charge. In other words, FPTC has a better planar molecular configuration, which, in turn, is conducive to intrinsic ICT interactions, resulting in a narrower band gap, which is consistent with absorption spectrum observations.

In Fig. 3, although the SMAs r-electrons in the HOMO are highly delocalized throughout the molecular skeleton, which provides efficient orbital interactions, the $\pi$-electrons in the LUMO are insufficiently delocalized at the terminal electron-deficient TC unit. This is mainly due to the large dihedral angle between the acceptor moiety and the phenyl group, which hinders the effective transfer of intramolecular charge, thus affecting the separation of photoelectron. By using DFT calculation, the HOMO/LUMO energy levels of FPTC and CPTC are $-4.43 /-3.67$ and $-4.37 /-3.65 \mathrm{eV}$, respectively. Most importantly, although the predicted values of the HOMO/LUMO energy levels and are not very accurate, the predicted value trend is consistent with the trend of the value calculated from the ground state absorption. 


\subsection{Photovoltaic properties}

In order to further evaluate the photovoltaic performance of these SMs, the solution-processable BHJ-SMOSCs devices were fabricated using FPTC and CPTC as the acceptor and PTB7-Th as the donor, which is an effective electron transport material and has an energy level that matches most acceptors and a complementary absorption spectrum. The typical inverted OSC devices with an architecture of glass/indium tin oxide (ITO) /zinc oxide (ZnO) / PTB7-Th: FPTC (CPTC)/ $\mathrm{MoO}_{3} /$ Ag were fabricated and its performance were characterized ${ }^{25}$. Then, we control different manufacturing device conditions to continuously optimize performance, such as controlling the thickness of the active layer, adjusting the mass ratio of donor (D): acceptor (A), different solvent additives, and thermal annealing. In consequence, the optimal device manufacturing conditions were obtained, whose conditions are D: A weight ratio of $1: 1.2$, thickness of $100 \mathrm{~nm}$, total solid content of chlorobenzene solution of $15 \mathrm{mg} \mathrm{L}^{-1}$ and the spin-coating rate of $2000 \mathrm{rpm}$, respectively. All of these SMs based blends have the same optimal thermal annealing temperature of $100^{\circ} \mathrm{Cat}$ the presence of $0.3 \%$ 1,8-diiodooctane (DIO) additive under AM 1.5G simulated solar with 100 light intensity in the ambient atmosphere. The current density-voltage $(\mathrm{J}-\mathrm{V})$ characteristics and external quantum efficiency (EQE) spectra of FPTC- and CPTC-based SM-OSCs are illustrated in Fig. $4 a-4 b$ and its corresponding parameters are shown in Table 2. In contrast, it was found that the FPTCbased devices containing fluorene as acceptor unit exhibited more excellent photovoltaic performance than CPTC-based devices whose donor unit is carbazole.

The device with FPTC: PTB7-Th shows a PCE of $0.87 \%$ with a $J s c$ of $3.42 \mathrm{~mA} / \mathrm{cm}^{2}$, a $V_{o c}$ of $0.71 \mathrm{~V}$ and an FF of $36.19 \%$ without any optimization. Through further optimization of thermal annealing and other conditions, the performance of the device was further improved, and a relatively high PCE of $1.09 \%$ with a $J s c$ of $4.21 \mathrm{~mA} / \mathrm{cm}^{2}$, a $V_{\text {oc }}$ of $0.72 \mathrm{~V}$ and an FF of $36.81 \%$ were obtained. However, the CPTC based BHJSM-OSCs shows slightly inferior PCE of $0.05 \%$ with a $V_{o c}$ of $0.49 \mathrm{~V}$, a $J_{s c}$ of $0.42 \mathrm{~mA} \mathrm{~cm}^{-2}$ and an FF of 0.24 . By continuously optimized, its photovoltaic performance has been improved very little with a PCE of $0.12 \%$. In comparison, the photovoltaic performance of FPTC-based devices is generally higher than that of CPTC-based devices. For example, the $V_{o c}$ of FPTC and the PCE is about $47 \%$ and $1 \%$ higher than that of CPTC, respectively. Particularly, the $J_{s c}$ of FPTC-based devices is 10 times that of CPTC-based devices. The CPTC-based BHJ-SM-OSC exhibits relatively poor photovoltaic performance whose reason may be that the carbazole unit has a stronger electron-donating capacity than fullerene moiety, which destroys the balance in the molecule to a certain extent, and the carbazole between the $\pi$ bridge or terminal groups are not greatly conjugated and form a larger dihedral angle. Therefore, in the process of preparing the film, too much intermolecular aggregation will be inevitably occurred, which is not conducive to intramolecular charge transfer. These results are completely consistent with the results of UV-vis absorption spectra and molecular calculations. In other words, the device containing fullerene group has more potential to be applied to the construction of high-efficiency organic small molecule solar cells.

In order to further evaluate the accuracy of photovoltaic performance measurement, the external quantum efficiency (EQE) spectra of the optimal FPTC: PTB7-Th and CPTC: PTB7-Th BHJ-SM-OSCs are 
shown in Fig. 4b. Apparently, the EQE curves have a similar trend to that of the $J_{s c}$ and all devices showed a broad solar spectral response ranging from 300 to $800 \mathrm{~nm}$. Compared to CPTC-based device, it could be seen that the EQE value of FPTC-based device are higher with a maximum EQE value of $19 \%$ in the whole spectral range, which is around four times of the CPTC device. The lower EQE of PTB7-Th: FPTC suggests that there is an exciton dissociation and charge transfer barrier or a severe charge recombination in the process of photocurrent generation. The $J_{s c}$ of the devices calculated from integration of the EQE spectra with a $1.5 \mathrm{G}$ reference spectrum are $3.58 \mathrm{~mA} \mathrm{~cm}-2$ and $0.44 \mathrm{~mA} \mathrm{~cm}^{-2}$ respectively, which is almost consistent with the measured $J_{s c}$ value, and the error is less than $5 \%$.

Efficient charge transport matters the prosperity of BHJ-OSCs and anticipates the device performance. Therefore, to further investigate the charge extraction and exciton dissociation properties between the PTB7-Th: FPTC and PTB7-Th: CPTC blend systems, the space charge limited current (SCLC) method was utilized to measure the charge transport properties. The hole-only device utilizes a structure of ITO/PEDOT: PSS/PTB7-Th: acceptors/Au, while the electron-only device adopts an Al/PTB7-Th: acceptors/Al configuration. The results of hole and electron mobilities are shown in Fig. $4 \mathrm{c}$ and $4 \mathrm{~d}$ (Supporting Information) and the detail data are listed in Table 2. The PTB7-Th: FPTC based device shows a hole and electron mobility of $1.69 \times 10^{-4} \mathrm{~cm}^{2} \mathrm{~V}^{-1} \mathrm{~s}^{-1}$ and $2.28 \times 10^{-4} \mathrm{~cm}^{2} \mathrm{~V}^{-1} \mathrm{~s}^{-1}$, respectively. However, compared to PTB7-Th: FPTC based device, the PTB7-Th: CPTC based device is a little bit worse with the value of $0.54 \times 10^{-4} \mathrm{~cm}^{2} \mathrm{~V}^{-1} \mathrm{~s}^{-1}$ and $0.62 \times 10^{-4} \mathrm{~cm}^{2} \mathrm{~V}^{-1} \mathrm{~s}^{-1}$, respectively. Obviously, the device containing fluorene moiety has higher and balanced charge mobility than their carbazole-containing counterparts, which reflects better device performance.

\subsection{Morphological analysis}

To further investigate the surface morphology of the morphology of PTB7-Th: FPTC and PTB7-Th: CPTC blend films, the tapping-mode atomic force microscopy (AFM) measurements were utilized and the corresponding height image and phase image were shown in Fig. 5 . It is known that the nanoscale morphology of active layer is highly important for high exciton dissociation and effective generation of free charge carriers. As shown in Figure 5a-d, the fluorene and carbazole based blend films exhibited rootmean-square (RMS) roughness of $4.57 \mathrm{~nm}$ and $5.42 \mathrm{~nm}$, respectively, indicating that the active fluorenecontained layers is relatively smoother than the other. Moreover, in the phase images, the blend thin film of shows PTB7-Th: FPTC shows relatively large dark domains and sizes of light that correspond to aggregations of carbazole-contained acceptor and PTB7-Th, which means that these domain sizes are not beneficial for effective exciton dissociation in the active layer. On the contrary, the blend film with FPTC showed slightly better phase separation with uniform interpenetrating network structures, which decreases the geminate recombination and bimolecular recombination, resulting in an improved hole and electron mobilities. Considering both AFM images and RMS roughness values, PTB7-Th: FPTC and PTB7Th: CPTC blend films exhibited similar and reasonable morphology, which is consistent with the abovementioned characterization results. 


\section{Conclusion}

In summary, two D-п-A-п-D small molecules, FPTC and СPTC with the same electron-withdrawing terminal acceptor group and $\pi$-conjugated bridge, but different electron-donating groups as the central core (fluorene, carbazole), have been designed, synthesized, and utilized to fabricate efficient solutionprocessable SMOSCs as acceptors materials. Both compounds were synthesized through only two procedures with high yields and all of them have great solubility in common organic solvents. Also, in this work, we utilized a great deal of method to characterized these SMs structure, photovoltaic and photoelectric properties, which could deeply understand the structure - property relationships and provide strategy to construct this type compound. Finally, we find that this FPTC: PTB7-Th device has a wider absorption spectrum and a molar extinction coefficient nearly four times higher than that of the CPTC: PTB7-Th device, a higher initial oxidation potential, and a lower onset reduction potential. Also, the higher electron mobility rate and hole mobility rate contributes to its better performances than CPTC, and the PCE of this device based on FPTC: PTB7-Th is nearly $1 \%$ higher than that of CPTC: PTB7-Th device, reaching up to $1.09 \%$ with a $V_{O C}$ of $0.71 \mathrm{~V}, \mathrm{a} J_{S C}$ of $3.42 \mathrm{~mA} \mathrm{~cm}^{-2}$. Moreover, it's convinced that there are many great features such as low-cost raw material, easy accessible of target compound, broad absorption bands reached near-infrared region, high extinction coefficients, good film-forming ability, suitable LUMO/HOMO levels, which implies that fluorene based small molecules are a potential candidate for photovoltaic applications and continuous development.

\section{Declarations}

\section{Acknowledgements}

This work was financial supported by Natural Science Foundation of Guangdong Province (2018A030313304), Talent Research Start-up Fund of Guangdong University and Technology (GC300501146), Dongguan Social Science and Technology Development Project (20185071401607) and Guangdong Public Welfare Fund Project (2017A010103047).

\section{References}

1. Chung C-L, Chen C-Y, Kang H-W, Lin H-W, Tsai W-L, Hsu C-C, Wong K-T (2016) A-D-A type organic donors employing coplanar heterocyclic cores for efficient small molecule organic solar cells. Org Electron 28:229-238

2. Hachi M, Slimi A, Fitri A, ElKhattabi S, Benjelloun AT, Benzakour M, McHarfi M (2019) New small organic molecules based on thieno[2,3-b]indole for efficient bulk heterojunction organic solar cells: a computational study. Mol Phys 118:(8)

3. Yao H, Chen Y, Qin Y, Yu R, Cui Y, Yang B, Li S, Zhang K, Hou J (2016) Design and Synthesis of a Low Bandgap Small Molecule Acceptor for Efficient Polymer Solar Cells. Adv Mater 28(37):8283-8287 
4. Lee J, Singh R, Sin DH, Kim HG, Song KC, Cho K (2016) A Nonfullerene Small Molecule Acceptor with 3D Interlocking Geometry Enabling Efficient Organic Solar Cells. Adv Mater 28(1):69-76

5. Tao R, Umeyama T, Kurotobi K, Imahori H, Effects of alkyl chain length and substituent pattern of fullerene bis-adducts on film structures and photovoltaic properties of bulk heterojunction solar cells. ACS Appl Mater Interfaces 2014, 6 (19), 17313-22

6. Faist MA, Shoaee S, Tuladhar S, Dibb GFA, Foster S, Gong W, Kirchartz T, Bradley DDC, Durrant JR, Nelson J (2013) Understanding the Reduced Efficiencies of Organic Solar Cells Employing Fullerene Multiadducts as Acceptors. Advanced Energy Materials 3(6):744-752

7. Huang W, Gann E, Chandrasekaran N, Prasad SKK, Chang S-Y, Thomsen L, Kabra D, Hodgkiss JM, Cheng Y-B, Yang Y, McNeill CR, Influence of Fullerene Acceptor on the Performance, Microstructure, and Photophysics of Low Bandgap Polymer Solar Cells. Advanced Energy Materials 2017, 7 (11)

8. Mumyatov AV, Goryachev AE, Prudnov FA, Mukhacheva OA, Sagdullina DK, Chernyak AV, Troyanov SI, Troshin PA, Monocyclopropanated fullerene derivatives with decreased electron affinity as promising electron acceptor materials for organic solar cells. Synthetic Metals 2020, 270

9. Lin Y, Zhan X, Non-fullerene acceptors for organic photovoltaics: an emerging horizon. Materials Horizons 2014, 1 (5)

10. Lin Y, Zhao F, Wu Y, Chen K, Xia Y, Li G, Prasad SK, Zhu J, Huo L, Bin H, Zhang ZG, Guo X, Zhang M, Sun Y, Gao F, Wei Z, Ma W, Wang C, Hodgkiss J, Bo Z, Inganas O, Li Y, Zhan X, Mapping Polymer Donors toward High-Efficiency Fullerene Free Organic Solar Cells. Adv Mater 2017, 29 (3)

11. Long $X$, Ding Z, Dou C, Liu J, Wang L, A double B $\leftarrow N$ bridged bipyridine (BNBP)-based polymer electron acceptor: all-polymer solar cells with a high donor: acceptor blend ratio. Materials Chemistry Frontiers 2017, 1 (5), 852-858

12. $<140$.pdf\&gt

13. Gautam P, Misra R, Koukaras EN, Sharma A, Sharma GD (2015) Donor-acceptor-acceptor-donor small molecules for solution processed bulk heterojunction solar cells. Org Electron 27:72-83

14. <fluorene11.pdf\&gt

15. Knaapila M, Monkman AP (2013) Methods for controlling structure and photophysical properties in polyfluorene solutions and gels. Adv Mater 25(8):1090-1108

16. Kozma E, Mróz W, Andicsová Eckstein A, Lukeš V, Galeotti F, Šišková A, Danko M, Catellani M, A joint experimental and theoretical study on the electro-optical properties of 1,6- and 1,7-fluorenyl disubstituted perylene diimide isomers. New Journal of Chemistry 2018, 42 (2), 1061-1066

17. Ma G, Zhao H, Wang J, Le Y, Jiang H, Deng H, Hao J, Wan W (2018) Studies of fluorine auxochrome in C9-fluorenyl anthracenes on optoelectronic property for blue electroluminescent materials. Dyes Pigm 158:420-427

18. Yahya M, Çakmaz D, Achelle S, le Gall E, Şahin E, Seferoğlu Z, Synthesis, photophysical, thermal properties and X-Ray studies of novel organic dyes bearing Inden-1-ylidene and fluorene. Journal of Photochemistry and Photobiology A: Chemistry 2021, 416 
19. Nielsen CB, Holliday S, Chen HY, Cryer SJ, McCulloch I, Non-fullerene electron acceptors for use in organic solar cells. Acc Chem Res 2015, 48 (11), 2803-12

20. He Q, Shahid M, Jiao X, Gann E, Eisner FD, Wu T, Fei Z, Anthopoulos TD, McNeill CR, Heeney M (2020) Crucial Role of Fluorine in Fully Alkylated Ladder-Type Carbazole-Based Nonfullerene Organic Solar Cells. ACS Appl Mater Interfaces 12(8):9555-9562

21. Ma D, Feng S, Zhang J, Kou C, Gong X, Li Q, Xu X, Yan S, Bo Z (2017) Non-fullerene small molecular acceptors with a carbazole core for organic solar cells with high open-circuit voltage. Dyes Pigm 146:293-299

22. Kim J, Kim YR, Kim M, Yoon CJ, Piao J, Jin JS, Sung JY, Kim H, Lee K, Suh H, Effect of conjugated small molecular electrolytes based on carbazole with $\mathrm{N}$ and $\mathrm{F}$ atoms for the automatic formation of electron transport layer in polymer solar cell. Synthetic Metals 2021, 275

23. Sylvianti N, Kim YH, Marsya MA, Kim DG, Kim JH, A-п-D-п-A type oligomer based on carbazole and benzothiadiazole for organic solar cells. Molecular Crystals and Liquid Crystals 2017, 655 (1), 166172

24. Janprapa N, Vchirawongkwin V, Kritayakornupong C, Influence of end-capped group on structural

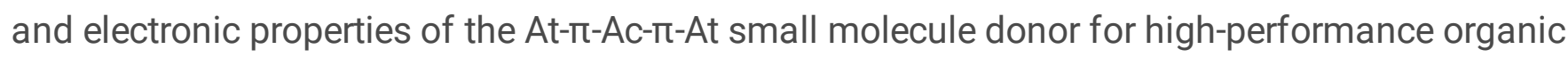
solar cells. Structural Chemistry 2020, 32 (1), 367-377

25. Anctil A, Babbitt CW, Raffaelle RP, Landi BJ, Material and energy intensity of fullerene production. Environ Sci Technol 2011, 45 (6), 2353-9

\section{Tables}

Please see the supplementary files section to view the tables.

\section{Figures}

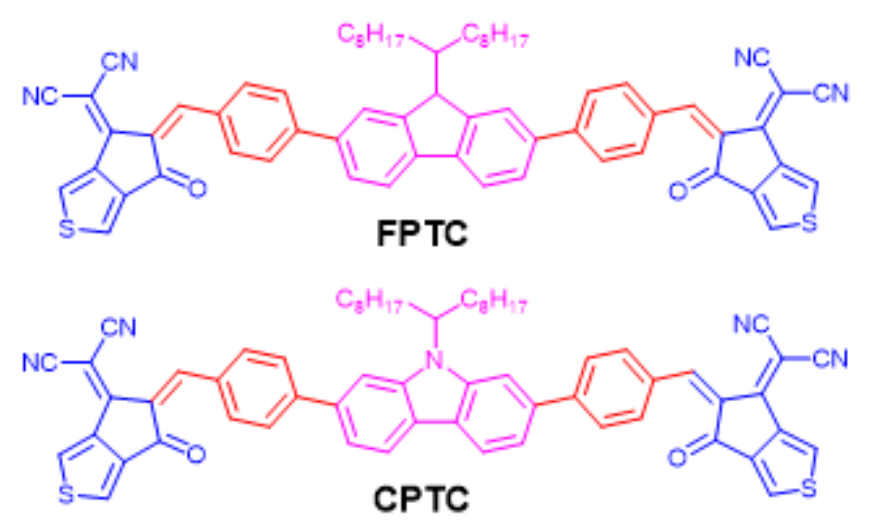

\section{Figure 1}

Molecular structures of FPTC and CPTC 

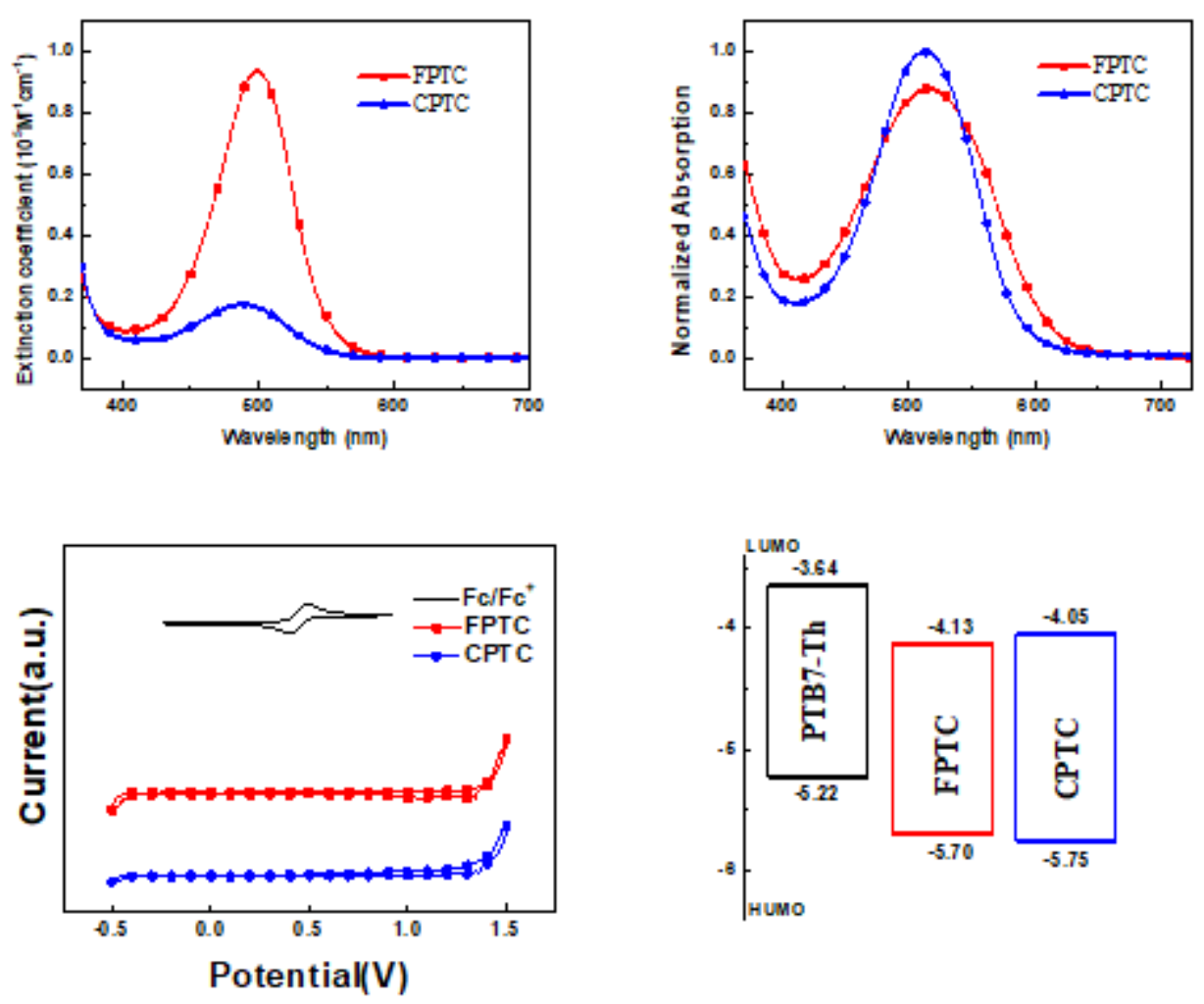

\section{Figure 2}

(a) UV-vis absorption spectra of FPTC and CPTC in chloroform solution. (b) Normalized UV-vis absorption spectra of FPTC and CPTC in films. (c) Cyclic voltammograms of FPTC and CPTC in $\mathrm{CH} 3 \mathrm{CN} / 0.1 \mathrm{M}$ Bu4NPF6. (d) Energy diagram relative to the vacuum level. 


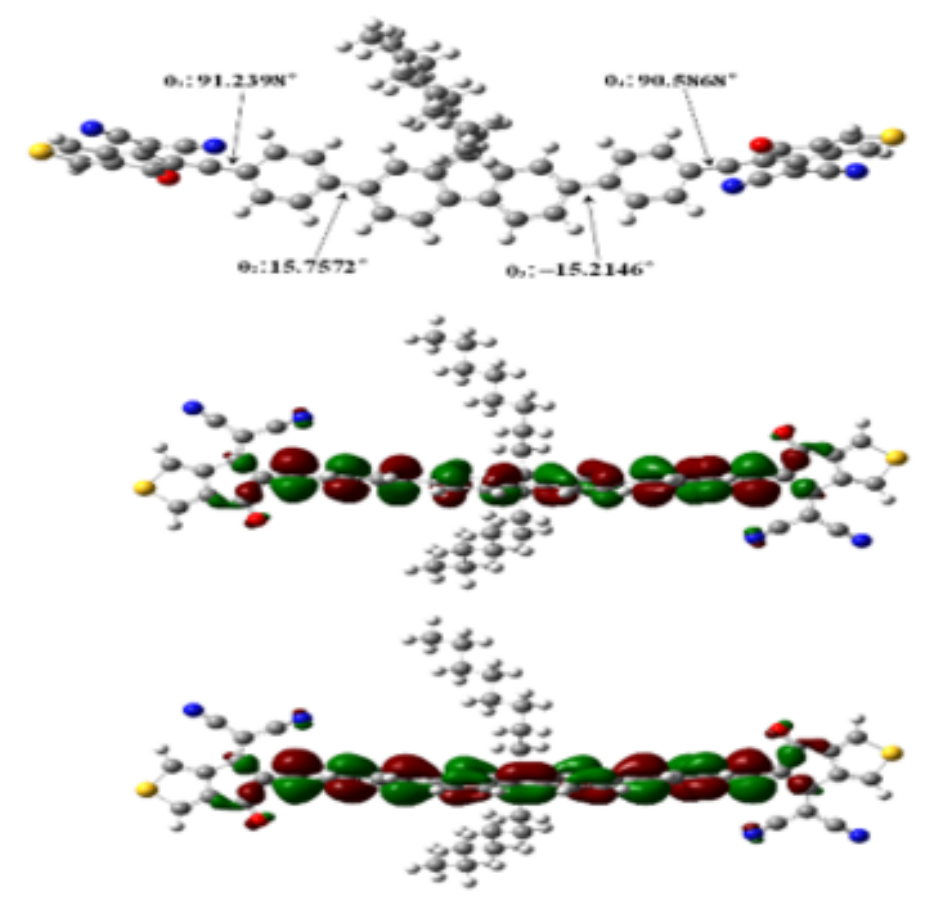

LUMO $-3.672 \mathrm{eV} \quad$ IIOMO $-4.434 \mathrm{eV}$

FPTC
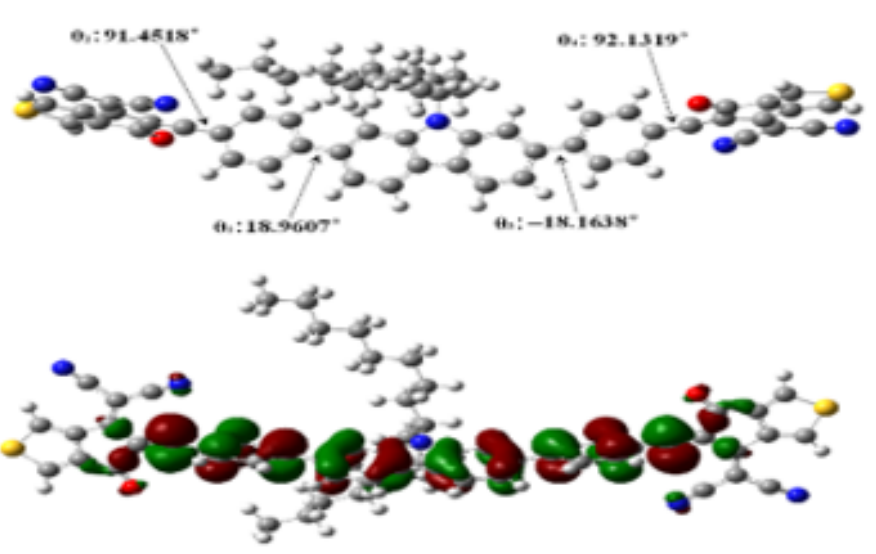

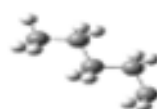

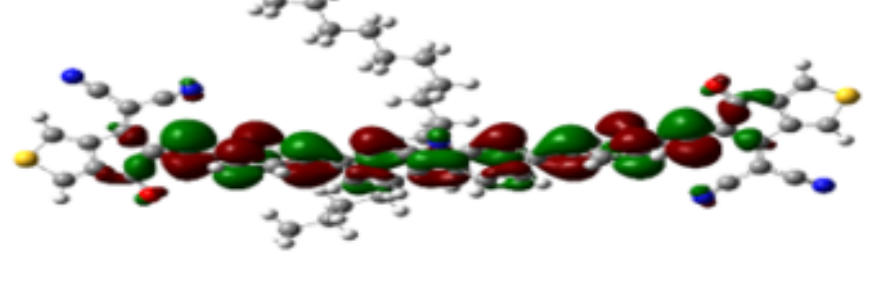

LUMO $-3.653 \mathrm{eV} \quad$ IIOMO $-4.365 \mathrm{eV}$

CРTC

Figure 3

The optimum geometry and electron-state-density distributions of HOMO and LUMO of FPTC and CPTC.
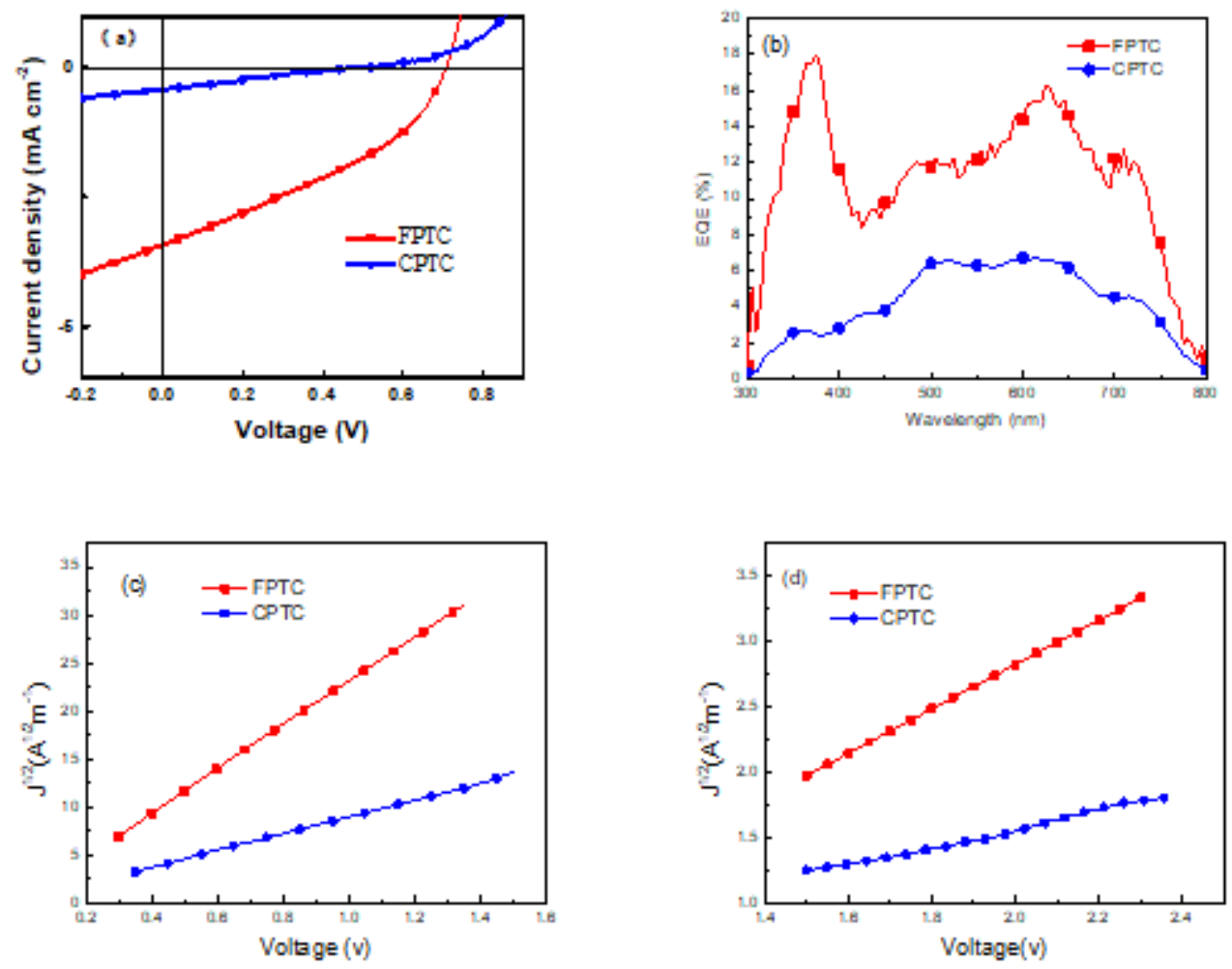

Figure 4 
(a) J-V curves (b)EQE spectra of FPTC or CPTC: PTB7-Th BHJ-SM-OSCs. (c)The hole mobilities of the PTB7-Th: acceptors blends. (d) The electron mobilities of the PTB7-Th: acceptors blends.
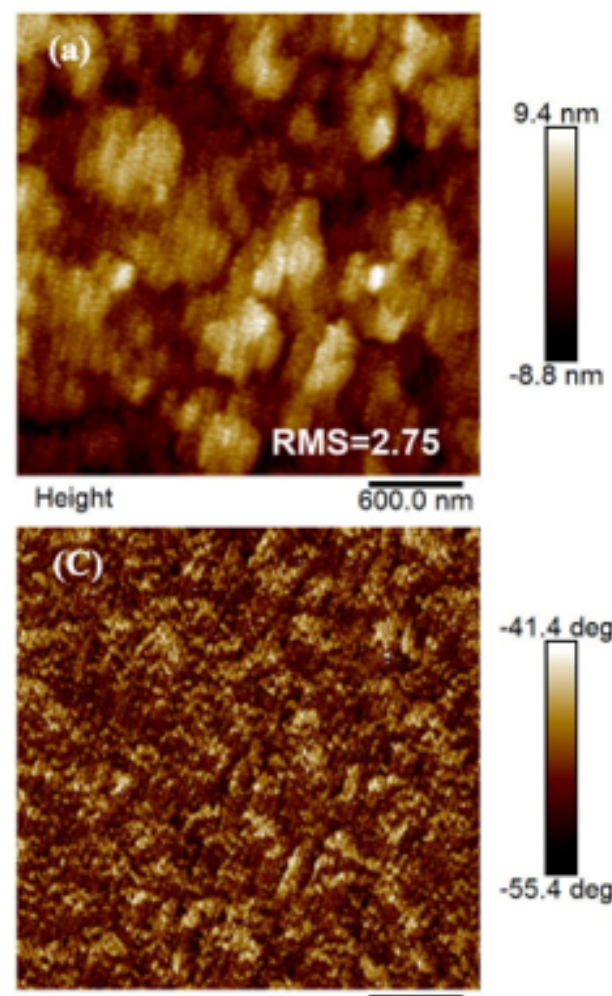

Phase

$6 \overline{00.0 \mathrm{~nm}}$

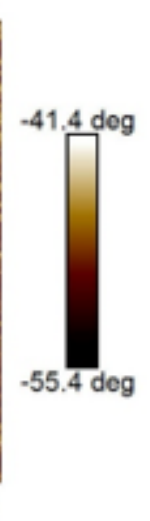

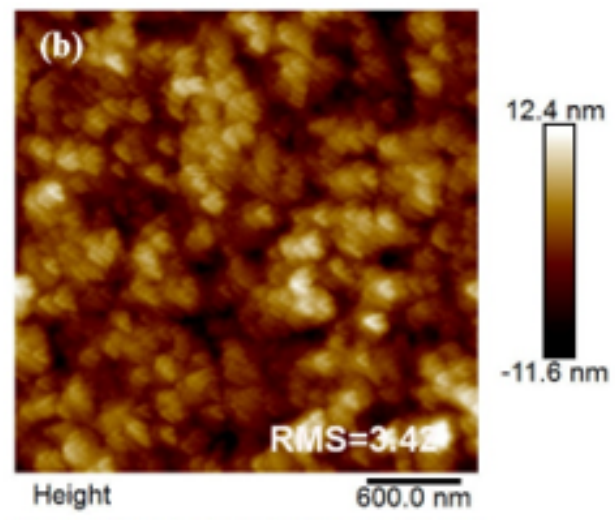

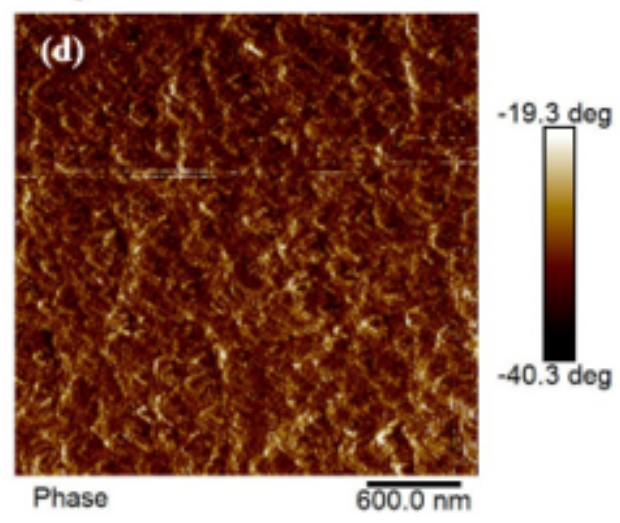

Figure 5

Tapping-mode AFM morphology images of the active layers of the PTB7-Th: FPTC, (a, c) PTB7-Th: CPTC, (b, d) blend films.

\section{Supplementary Files}

This is a list of supplementary files associated with this preprint. Click to download.

- Tables.docx

- Scheme1.docx

- ResearchHighlights.doc

- SupportingInformation.docx 